\title{
Creation of Patient-Specific Silicone Cardiac Models with Applications in Pre-surgical Plans and Hands-on Training
}

\author{
Megan S. Mattus ${ }^{1}$, Tate B. Ralph ${ }^{1}$, Sister M. Pieta Keller ${ }^{1}$, Alexa L. Waltz ${ }^{2}$, Matthew T. Bramlet ${ }^{2}$ \\ ${ }^{1}$ Jump Simulation, OSF HealthCare ${ }^{2}$ Advanced Imaging and Modeling Lab, OSF HealthCare Jump Trading Simulation \& Education Center, University of \\ Illinois College of Medicine
}

\section{Corresponding Author}

Alexa L. Waltz

alexa.I.waltz@osfhealthcare.org

\section{Citation}

Mattus, M.S., Ralph, T.B., Keller, S.M.P., Waltz, A.L., Bramlet, M.T. Creation of Patient-Specific Silicone Cardiac Models with Applications in Pre-surgical Plans and Hands-on Training. J. Vis. Exp. (180), e62805, doi:10.3791/62805 (2022).

\section{Date Published}

February 10, 2022

DOI

$10.3791 / 62805$

URL

jove.com/video/62805

\section{Abstract}

Three dimensional models can be a valuable tool for surgeons as they develop surgical plans and medical fellows as they learn about complex cases. In particular, 3D models can play an important role in the field of cardiology, where complex congenital heart diseases occur. While many 3D printers can provide anatomically correct and detailed models, existing 3D printing materials fail to replicate myocardial tissue properties and can be extremely costly. This protocol aims to develop a process for the creation of patient-specific models of complex congenital heart defects using a low-cost silicone that more closely matches cardiac muscle properties. With improved model fidelity, actual surgical procedural training could occur in advance of the procedure. Successful creation of cardiac models begins with the segmentation of radiologic images to generate a virtual blood pool (blood that fills the chambers of the heart) and myocardial tissue mold. The blood pool and myocardial mold are 3D printed in acrylonitrile butadiene styrene (ABS), a plastic dissolvable in acetone. The mold is assembled around the blood pool, creating a negative space simulating the myocardium. Silicone with a shore hardness of $2 \mathrm{~A}$ is poured into the negative space and allowed to cure. The myocardial mold is removed, and the remaining silicone/ blood pool model is submerged in acetone. The described process results in a physical model in which all cardiac features, including intra-cardiac defects, are represented with more realistic tissue properties and are more closely approximated than a direct 3D printing approach. The successful surgical correction of a model with a ventricular septal defect (VSD) using a GORE-TEX patch (standard surgical intervention for defect) demonstrates the utility of the method.

\section{Introduction}


Nearly 1 in 100 children in the United States are born with congenital heart defects (CHD). Due to the propensity of mothers with CHDs to have children with CHDs, there is an expectation that the rate may more than double over the next seven generations ${ }^{1}$. While not each $\mathrm{CHD}$ is considered complex or severe, the general growth expectation indicates that there is motivation to improve the technology and procedures capable of addressing CHD treatment. As technology improves, cardiac surgeons often express a willingness to tackle more complex procedures. This willingness has led to an increased number of complex cardiac procedures, driving the need for more advanced techniques of surgical planning and education. In turn, this leaves cardiac surgeons in need of highly accurate, patientspecific models and cardiac surgical fellows in need of highly effective training methods.

Congenital cardiac surgery is one of the most technically demanding surgical disciplines due to the small size of the patients, the complexity of the cardiac abnormalities, and the rarity of some abnormalities ${ }^{2}$. In the most extreme cases, a child may be born with a single ventricle. It is not uncommon for the surgeon to take a vessel $2.0 \mathrm{~mm}$ in diameter and patch it with fixed pericardium to create a $1.0 \mathrm{~cm}$ vessel allowing a newborn to grow in this life-saving procedure all while under the clock, as the newborn is in complete circulatory arrest. Between the normal four-chamber heart and these extreme examples are innumerable possibilities of chamber size and valve positions that constitute highly complex 3D puzzles. The role of the congenital cardiac team is to clearly delineate the unique anatomy and develop a plan to reconfigure the organic tissue into a functional heart that will allow a child to grow with the best chance at a normal life. Accurate models allow for deliberate surgical practice and repetition in an environment where errors can be forgiven and will not result in patient harm ${ }^{3,4}$. This training leads to the development of improved surgical expertise, as well as technical and judgment skills. However, limited resources and the rarity of certain cardiac conditions can make achieving the desired level of repetition and visualization nearly impossible. To help account for this resource deficiency, there has been an increase in the utilization of simulations for education ${ }^{2,3}$. Commonly utilized simulation or modeling techniques include human cadavers, animal tissues, virtual reality models (VR), and 3D printed models.

Cadaveric tissue has historically been regarded as the gold standard for surgical simulation, with animal tissue a close second. Cadavers and animal tissues can produce high fidelity simulations because they contain the anatomical structure of interest, all surrounding tissues, and allow for perfusion techniques to simulate blood flow ${ }^{4}$. Despite the benefits of tissue models, there are downsides. Embalmed tissue experiences reduced mechanical compliance, making some operations unrealistic and difficult to perform. Tissues require constant maintenance, specific facilities, are not reusable ${ }^{2}$, can be costly to obtain ${ }^{3}$, and have historically been the subject of ethical concerns. Most significantly, congenital cardiac conditions are simply not available in cadaveric samples.

VR and 3D printed models $5,6,7,8,9,10$ provide another option for cardiac education, simulation, and modeling to aid in the creation of pre-operative plans. These models reduce ambiguity associated with a user's varied visuo-spatial ability to interpolate $2 D$ images as a $3 D$ structure ${ }^{10,11}$. The virtual environment can contain surgical tools that can be manipulated and interact with models, allowing surgeons and fellows to develop hand-eye coordination, fine motor skills, and familiarity with some procedures ${ }^{4}$. Current popular 3D 
printing technologies, including fused deposition modeling (FDM), stereolithography (SLA), selective laser sintering (SLS), and polyjet have been found to produce models with submillimeter precision ${ }^{13}$. Both VR and 3D printed models are reusable and can be extremely detailed; models can even be generated from patient radiologic imaging data, resulting in replicas of patient anatomy. Despite the many benefits of a VR or 3D printed models, they fall short when the cost and haptic fidelity requirements of congenital heart surgery are considered. The setup of a VR environment has a high cost, and VR environments cannot provide real-world haptic feedback. While haptic fidelity technology is improving, the current gap inhibits a student's ability to become familiar with the fine-motor skills necessary to perform procedures ${ }^{4}$. Similarly, depending on the type of 3D printing technology used, the cost of 3D printing can be quite high, as the printer purchase price and print material cost must be considered $^{11,14}$. A single high-fidelity cardiac model with realistic haptic feedback can be produced using a high-end printer but will cost hundreds of dollars in material alone with a printer purchase price over 100,000 USD $^{15}$. A cardiac model produced using a filament with a shore hardness of 26-28 A was found to cost approximately 220 USD per model ${ }^{16}$. Alternatively, many low-cost 3D printers and technologies are available that have a printer purchase price of less than 5,000 USD. Average material prices for a cardiac model generated on a low-cost FDM printer was found to be about 3.80 USD using a material with a shore hardness of $82 \mathrm{~A}$ and 35 USD using a material with a shore hardness of 95 $A^{15,16}$. While these machines do offer a low-cost solution, it comes at the cost of haptic fidelity.

While VR and 3D printing can allow for detailed visual and conceptual evaluation of a cardiac condition, the high price associated with producing a model for hands-on surgical simulation is often a significant barrier. One solution is the use of silicone to create a physically and texturally accurate cardiac model. Patient-specific silicone models can facilitate a deeper understanding of unique anatomy by allowing surgeons to see, feel, and even practice a procedure while experiencing realistic haptic feedback in an environment that involves minimal risk to a patient and has no consequences if the procedure is unsuccessful ${ }^{9}$. Silicone molding has been shown to be an effective method to model human anatomy that produces models with physical properties that are significantly closer to real tissue than models generated from low-cost $3 D$ printing ${ }^{17}$. Scanlan et al., compared the properties of low-cost 3D printed to silicone molded cardiac valves to evaluate similarity to real tissue; the study found that while the physical properties of the silicone valves were not an exact replica of real tissue, the properties were far superior to the $3 D$ printed valves ${ }^{17}$. The $3 D$ printing material used in the study is among the softest materials available for lowcost 3D printers and possesses a shore hardness between 26 and $28 A^{18}$. The platinum cure silicone recommended for use in the protocol below has a shore hardness of 2 A which is far closer to the shore hardness of cardiac tissue, 43 on the 00 scale, or approximately $0 A^{19,20}$. This difference is significant because the silicone models allow for highfidelity fine- motor skill training that the directly 3D printed materials do not achieve. The total material cost for the model proposed in this protocol is less than 10 USD. The proposed silicone models combine the soft tissue properties necessary for realistic haptic feedback with the versatility and precision of low-cost 3D printed models.

While the benefits of silicone may appear to make it the obvious choice for model creation, the use of silicone has been restricted by the anatomy that can be molded. Freshly mixed silicone is a liquid that requires a mold 
to hold it in the desired shape as it cures. Historically, silicone cardiac molds could only contain details of the outer surface of the model. Intra-cardiac details, including the entire blood pool region, would be filled with silicone and lost. Previous studies have achieved silicone models of specific areas of interest within the heart (e.g., aortic $\operatorname{root}^{21}$ ) or have used an extrapolatory method to simulate myocardial tissue $^{22}$. This protocol is novel as it seeks to combine the use of silicone material with high-resolution anatomical, full myocardial simulation- specifically avoiding any method of extrapolation. To our knowledge, no descriptive manuscript has provided a methodology combining these aspects. The method described in this protocol introduces a technique to achieve a patient-specific cardiac model with intracardiac anatomic replication accurate enough for surgical preoperative practice. The method involves the creation of a myocardial mold to hold the silicone in the proper shape as it cures and an inner mold to preserve the internal, intracardiac details of the model and prevent the silicone from filling the blood pool region of the heart. The inner mold must then be dissolved away, leaving an entire silicone cardiac model with patient-specific anatomy on the outer and inner surfaces. Without the proposed protocol of cardiac model creation herein, no low-cost solution exists to simulate the surgical procedure with a material that mimics the actual tissue characteristics of the myocardium.

\section{Protocol}

The protocol was completed in a manner corresponding to the best ethical practices of the author's institution including the proper handling of any patient information and the assurance of the required consents necessary to use patient specific data. When used, such data was anonymized to ensure the protection of the patient's private health information.
NOTE: The following protocol is written in a software neutral manner, as there are many different programs that can accomplish the various steps. For this particular case, Materialise Mimics Medical 24.0 was used for segmentation, and Materialise Magics was used for 3D manipulation and creation of the segmented models and cases. Specific instructions for those programs will be included in addition to the generalized approach.

\section{Segment patient anatomy}

1. Per SME, obtain a patient radiologic imaging dataset, typically a CT or MRI, acquired using a 3D protocol for adequate resolution. Open dataset in a computer aided design (CAD) segmentation software ${ }^{23}$.

1. Refer to the institution's radiology protocols for proper image acquisition (since each patient requires different considerations, it is difficult to provide a specific guideline). But as a representative example, these are the settings we used in a previously documented 3D model case: CT 3D protocol suggests parameters: slice scanner in axial mode, slice thickness and space between slices of $0.625 \mathrm{~mm}, \mathrm{Kv}$ of 70 , Smart $\mathrm{mA}$ range of $201-227$ (smart MA mode 226), rotation speed at 0.28 ms. MRI 3D protocol suggested parameters: slice scanner in axial mode, slice thickness and space between slices of $0.625 \mathrm{~mm}$.

2. Generate an initial segmentation of the myocardial tissue using a Hounsfield unit (HU) threshold tool with upper and lower limits set to values that are appropriate and specific to the dataset. Refine the selection as needed to accurately capture the anatomy. It is recommended to use tools with the following capabilities: crop, add 
and subtract, region grow, multi-slice edit, and cavity fill. In Mimics, right click in the Project Manager area and select New Mask. Adjust the mask in the generated dialogue box either with the pre-set anatomical windows provided, exact HU measurements, or by sliding the provided tool until the anatomy desired is masked by the tool.

3. Generate a segmentation of the blood pool. Utilize the steps described in step 1.2 to accomplish this. In Mimics, use the pre-set anatomical HU window of 226 to 3071 to capture the blood pool.

4. If the model being generated is intended for use in patient care, let a cardiologist, radiologist, or other subject matter expert (SME) review the virtual model segmentations before proceeding to the next step to ensure all anatomic features and defects were segmented accurately and will be present in the complete model.

5. Generate a myocardial case model by inverting the myocardium segmentation using a cavity fill tool in the empty space around myocardium segmentation and subtracting the blood pool segmentation from the inverted myocardium using a Boolean subtract tool. It is recommended to use a cavity fill tool, a Boolean tool, and the previously generated myocardium and blood pool segmentations to accomplish this. In Mimics, Cavity Fill $>$ Indicate spaces around myocardium mask. Next, use the Boolean tool and fill the provided dialogue to Minus the blood pool mask from the myocardium mask.

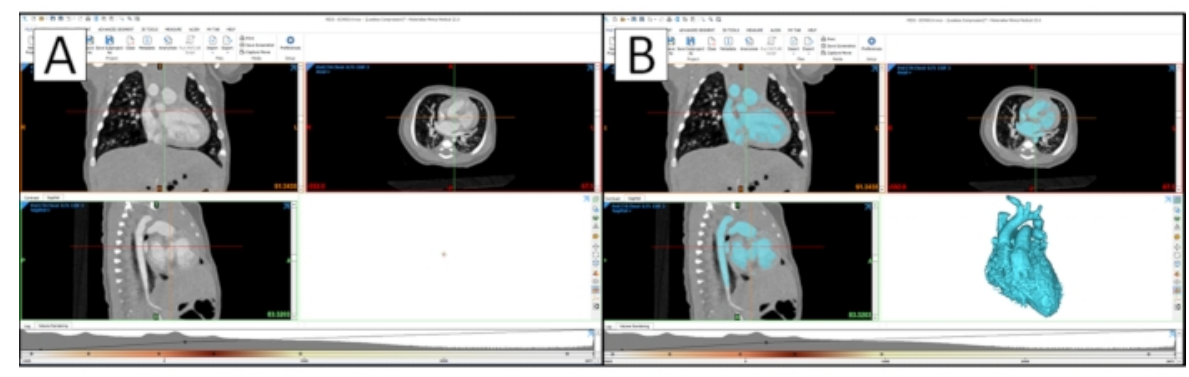

Figure 1: Cardiac segmentation in a CAD segmentation software. (A) Cardiac segmentation in CAD segmentation software with the raw patient image data. (B) Segmentation with 3D rendering of the blood pool model. Please click here to view a larger version of this figure.

6. View the $3 \mathrm{D}$ rendering of the final blood pool and myocardial case segmentations. Per SME suggestion and approval, remove any blood vessels from the 3D blood pool model that are not necessary for evaluation, comprehension, or repair of the target anatomy. In Mimics, select 3D Preview in the options next to the viewing window (defaulted to the bottom right view of the four-pane default view. Select the mask of interest in the Project Manager. To edit, select the Edit Mask tool. In the provided dialogue, select the Lasso tool and ensure that Remove is selected. This will allow editing of the actual 3D Preview of the mask.

NOTE: The editing tool is an infinite cut plane and will remove any part of the mask selected in the Z-direction. 
7. Generate 3D objects of the final blood pool and myocardial case segmentations. Smooth the 3D myocardial case model using a smooth object tool. Per SME suggestion and approval, adjust iteration and smooth factor parameters as necessary for the specific model to create a case model that is as smooth as possible but has not lost any important anatomical details.

8. Once approved by an SME, export the models in STL format for use in a 3D model editing software. In Mimics, right-click on a specific mask in the Project Manager $>$ Create Object. In the provided dialogue, ensure the Optimal setting is selected and click Okay.

9. Once the model is created, it will appear in the Object window, typically below the Project Manager window. From there, right-click on a generated model and select Smooth. Parameters for this case were five iterations at $0.4 \mathrm{~mm}$ smoothing.

10. Save/export the final 3D blood pool and myocardial case models as standard tessellation language (STL) files. Right-click the desired model $>$ STL $+>$ follow the provided dialogue to export an STL version of the model.

\section{Create the digital molds}

1. Open the myocardial case model STL file in a CAD program. It is recommended to render the visibility of the case in a transparent manner to allow the myocardium to be visible on the interior of the myocardial case mold. In Magics, import the STLs generated through Import Part. In the Project Management window, select the Transparent option of model rendering.

2. Trim excess myocardial case material from the model using a cut or punch tool. It is only necessary to have approximately $0.5 \mathrm{~cm}$ between the outer edge of the case and the myocardial imprint on the inner case wall. Additional material will add to the time needed to $3 \mathrm{D}$ print but will not affect the end product. In Magics, Cut > Indicate Polyline > select points of interest > Apply.

NOTE: The editing tool is an infinite cut plane and will remove any part of the mask selected in the Z-direction.

3. Cut the myocardial case into multiple pieces that will allow the case to be assembled around the complex anatomy of the blood pool mold. It is recommended to use a cut and/or punch tool to accomplish this.

NOTE: The following steps provide a suggestion of cuts to make in the myocardial case that will divide it into four sections that have been found to be sufficient for both diagnostic accuracy and case assembly around the blood pool for many cardiac models. However, each model will be different, making it vital to keep in mind that the case must be assembled around the blood pool before silicone is poured and removed after the silicone sets. Pay special attention to any locations where the case must pass through a loop in the blood pool or surround long blood vessels. Features such as these may require the myocardial case to be cut into additional pieces in the region where the feature exists to ensure assembly and disassembly around the blood pool will be possible.

4. Adjust the view of the myocardial case through rotation and panning tools to point the apex of the heart down and the aorta arch horizontal. Make a horizontal cut through the aorta that divides the myocardial case into a lower half that contains the apex and an upper half. Length of this cut and all subsequent cuts will vary with each cardiac model. In Magics, use the left and right mouse buttons to control rotation and panning, respectively. 
From there, Cut $>$ Indicate Polyline $>$ select points of interest > Apply.

NOTE: The editing tool is an infinite cut plane and will remove any part of the mask selected in the Z-direction.

1. Make a vertical cut along the widest section of the lower half of the myocardial case. Ensure that the lower half of the myocardial case is split roughly in half.

2. Make a second vertical cut along the widest section of the upper half of the myocardial case. Ensure that the upper half of the myocardial case is split roughly in half.
5. Add pegs (props) to the myocardial case pieces to ensure proper alignment during assembly. It is recommended to use a prop generation tool and a Boolean subtraction tool with a clearance value of $0.25 \mathrm{~mm}$ to create matching props and prop cavities. In Magics, Add Props > indicate position on model > Apply.

6. Create a $1.0 \mathrm{~cm}$ diameter silicone fill hole to one of the myocardial case upper half pieces. The myocardial surface features directly below the fill hole will be obscured, so ensure that the fill hole is not over any external anatomical features that will be vital to the use of the model. Verify hole placement with a SME.

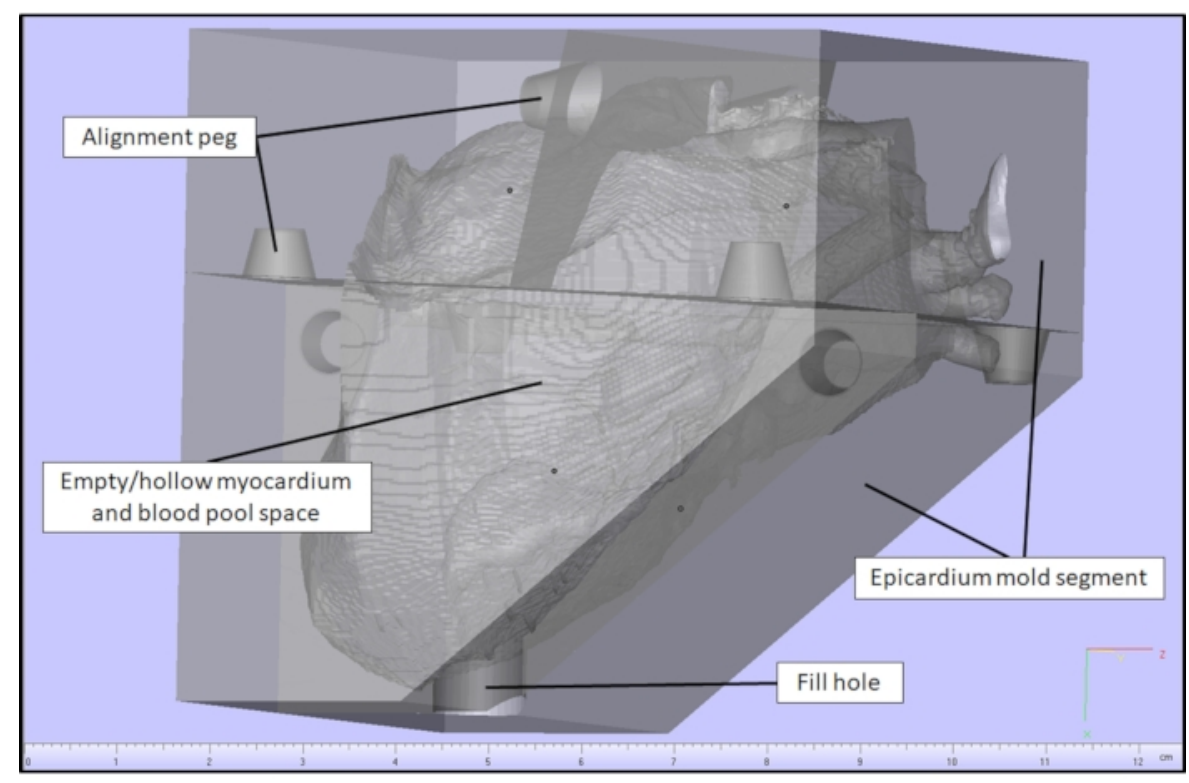

Figure 2: Myocardial case model in a CAD software. Myocardial case generated in a CAD software for a cardiac case with a VSD. Please click here to view a larger version of this figure.

7. Check diagnostics on all case pieces individually to ensure the following errors are not present: inverted normal, bad edges, bad contours, near bad edges, planar holes, or shells. If an error is detected, repair it using a fixing tool/wizard if available or manually if not available. In Magics, Check Diagnostics > Auto-resolve.

8. Fix errors that cannot be resolved manually or with a fixing tool/wizard with a part shrink wrap via a shrink wrap tool. Adjust shrink wrap sample interval and gap fill 
values as necessary to correct the errors on the specific piece without altering the physiology upon SME review. In Magics, Fix > Shrink Wrap > follow dialogue.

9. Save/export the individual myocardial case pieces as STL files.

\section{Create the physical molds}

1. Open the myocardial case and blood pool models in the appropriate slicer software to produce 3D printing files (G-Code file) for an additive manufacturing (AM) 3D printer. Arrange the pieces of the myocardial case using a rotate and/or lay flat tool, so any side that will meet up with another case piece is vertical. Add 3D print supports to all pieces manually or using an automatic support generation tool provided in the software, if available.

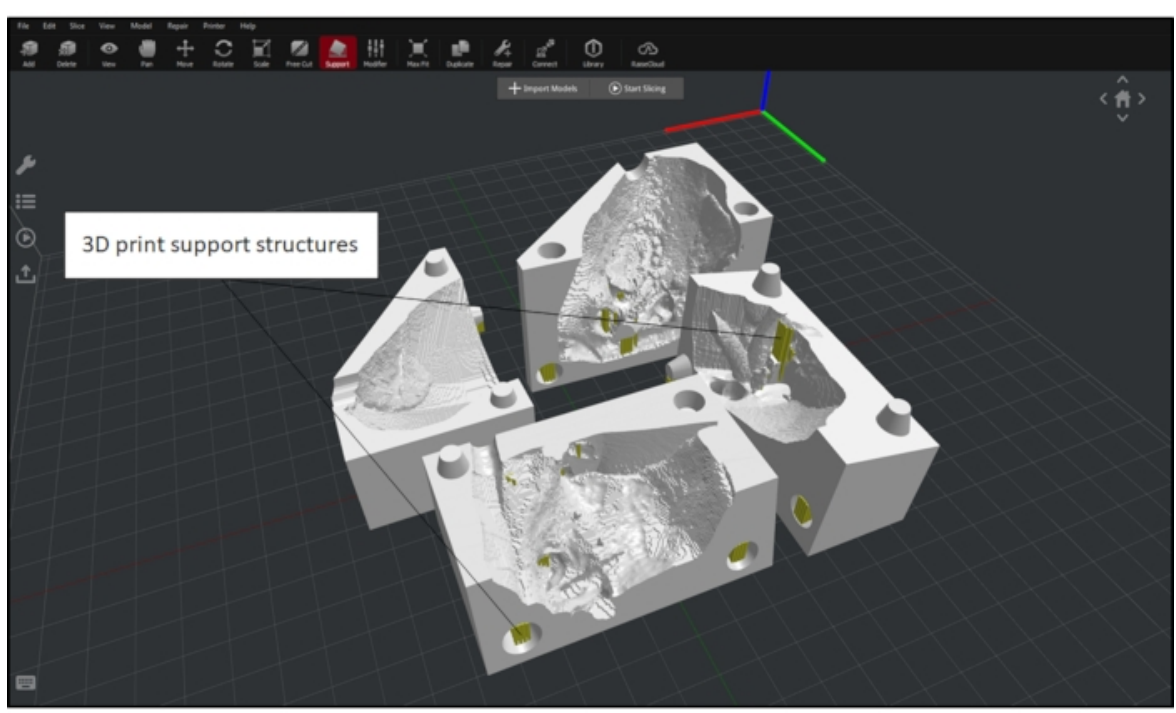

Figure 3: Myocardial case and blood pool setup in a 3D printing CAD software. Myocardial case and blood pool with proper orientation and added support in preparation for 3D printing in a 3D printing CAD software for a cardiac case with a VSD. Please click here to view a larger version of this figure.

2. Slice the models to generate G-code for use on the 3D printer with the following parameters: Blood pool in ABS using: heated bed temperature of $100^{\circ} \mathrm{C}$, extruder temperature of $250^{\circ} \mathrm{C}$, infill density of $5 \%$, default printing speed of $50 \mathrm{~mm} / \mathrm{s}$, inner shell speed of $70 \mathrm{~mm} / \mathrm{s}$, outer shell speed or $50 \mathrm{~mm} / \mathrm{s}$; Myocardial mold in ABS or polylactic acid (PLA) using: heated bed temperature of $60{ }^{\circ} \mathrm{C}$ for PLA or $100{ }^{\circ} \mathrm{C}$ for $\mathrm{ABS}$, extruder temperature of $205^{\circ} \mathrm{C}$ for PLA or $250{ }^{\circ} \mathrm{C}$ for ABS, infill density of $15 \%$, default printing speed of $50 \mathrm{~mm} / \mathrm{s}$, inner shell speed of $80 \mathrm{~mm} / \mathrm{s}$, and outer shell speed of $30 \mathrm{~mm} / \mathrm{s}$.

3. Save/export the G-code.

4. Upload the printing file to the $3 \mathrm{D}$ printer using a Flash Drive or Wi-fi connection, depending on the printer's capabilities, ensure correct filament is loaded onto 3D 
printer and start print. The 3D printer should meet the following requirements: compatible and equipped with a nozzle diameter less than $0.4 \mathrm{~mm}$ and capable of a layer resolution less than $0.25 \mathrm{~mm}$. Upon completion of printing, use needle nose pliers and tweezers to remove all support material from the printed pieces.

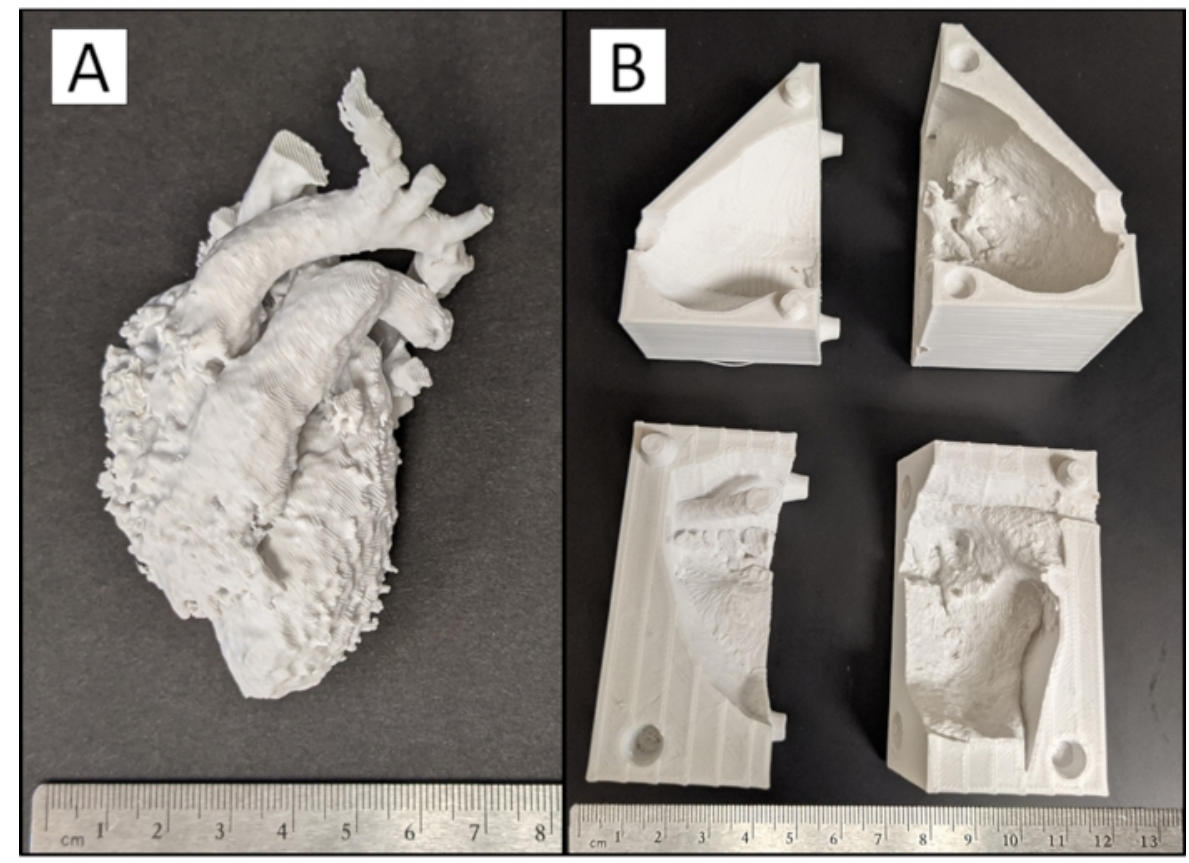

Figure 4: 3D printed model pieces. Photograph of $(\mathbf{A})$ physical blood pool and $(B)$ myocardial case pieces of a cardiac case with a VSD produced from the 3D printer with support material removed. Please click here to view a larger version of this figure.

5. Assemble the myocardial case pieces around the blood pool mold, ensuring all pieces fit together tightly. If the myocardial case cannot fit around the blood pool, make small adjustments to the case mold piece using a handheld rotary sanding tool to remove material. If a large adjustment is needed, it may be necessary to edit the STL file in a CAD software and create a new 3D print. CAUTION: Use eye protection when using a handheld rotary sanding tool. Use of a rotary sanding tool on the blood pool or myocardium case will cause the plastic to melt. Use sparingly and with caution.
NOTE: The protocol can be paused between any steps prior to this point.

6. Perform an acetone vapor smooth if the myocardial case was $3 D$ printed using $A B S$, and a smoother silicone surface finish is desired by the SME. If a smoother surface finish is not desired or needed, skip the vapor smooth process with minimal effect to the final anatomy of the model.

CAUTION: Acetone is volatile and flammable. Be sure to set up in a well-ventilated area away from open flames or sparks. Additionally, acetone will dissolve polyvinyl chloride (PVC) and polystyrene. If a plastic 
container is being used, ensure it does not contain PVC or polystyrene.

1. Line the bottom and sides of a container that will not be affected by acetone with paper towels. Pour the acetone on the bottom paper towel and allow it to diffuse up the paper towels on the side of the container but not form a pool in the bottom. The amount of acetone needed will vary depending on the size of the container used; here, $30 \mathrm{~mL}$ of acetone was used in a container with a base volume of approximately $400 \mathrm{~cm}^{3}$.

2. Place a piece of aluminum foil in the container to cover the bottom paper towel. Place the myocardial case pieces onto the aluminum foil and orient the myocardial pieces so that the faces desired to be smoothed are vertical. Ensure that the myocardial pieces are not touching one another or the paper towels on the wall of the container.
3. Place a lid on the container or cover with aluminum foil and allow the myocardial case pieces to remain undisturbed in the container until $\sim 80 \%$ of the desired surface finish is achieved, per visual inspection. The time needed to complete the vapor smooth process will vary depending on the size of the container and the amount of acetone used. Begin checking the myocardial case pieces for the desired surface finish at 15 min intervals after an initial 30 min. For this study, vapor smoothing took $2 \mathrm{~h}$ for a $150 \mathrm{~mL}$ structure.

4. Wearing gloves, carefully remove the myocardial case pieces from the container touching only the outer surfaces. Allow the pieces to completely degas in a well-ventilated area for $\sim 30$ min, or until smooth, dry, and hard.

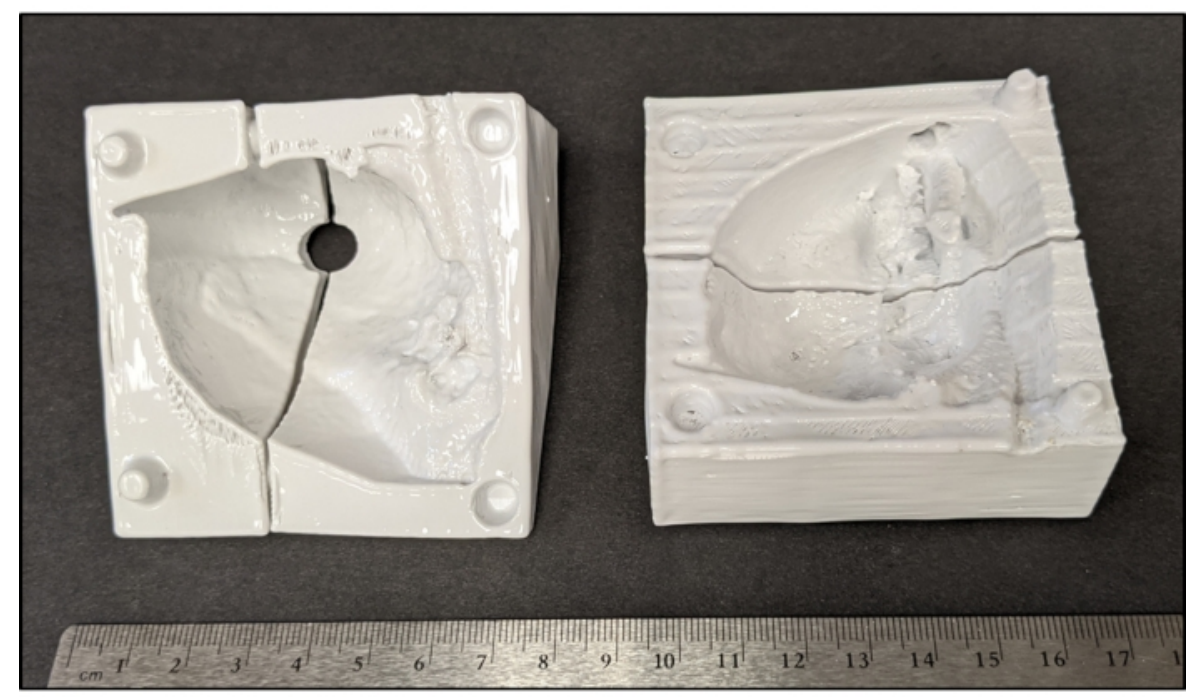

Figure 5: Vapor smoothed myocardial case pieces. Photography of myocardial case pieces of a cardiac case with a VSD after an acetone vapor smooth. Please click here to view a larger version of this figure. 


\section{Pour silicone}

NOTE: Some contaminants, including latex and sulfur, can inhibit the curing of silicones if they come into contact. It is advised to review any technical bulletins prior to attempting to use silicone.

1. Estimate the amount of a two-part platinum cure silicone that will be needed to create the myocardial model; the amount of silicone needed will vary depending on the size of the model being created. Alternatively, measure the volume of the myocardium segmentation using a CAD software to determine the amount of silicone needed. Ensure that the silicone possesses the following properties: shore hardness of $2 \mathrm{~A}$, tensile strength of $1,986 \mathrm{kPa}$, elongation at break of $763 \%$, shrinkage less than $0.0254 \mathrm{~mm} / \mathrm{mm}$, mixed viscosity of $18,000 \mathrm{cps}$, pot life of $12 \mathrm{~min}$, and cure time of $40 \mathrm{~min}$. This study required $300 \mathrm{~mL}$ of silicone.

2. Thoroughly agitate part $A$ and part $B$ of the silicone before pouring the necessary amounts, in the correct proportions, into a mixing cup. If color is desired on the model, add pigment and mix all parts and pigment thoroughly. For this study, $150 \mathrm{~mL}$ of both Part $\mathrm{A}$ and Part $B$ were mixed and agitated by hand or with an agitator. A Sil-pigment product color "blood" (see Table of Materials) was added by craft stick until the desired color was achieved.

3. Place the thoroughly mixed silicone into a vacuum chamber at $29 \mathrm{in} \mathrm{Hg}$ for 2-3 $\mathrm{min}$ to de-gas. The silicone will expand during the de-gassing process to approximately twice its volume, be sure the mixing container has enough room to allow for the expansion. Vent and remove de-gassed silicone from the chamber and submerge the blood pool in the silicone to thoroughly coat it, ensuring all voids and cavities in the blood pool are filled with silicone.

4. Thoroughly spray all pieces of the myocardial case with an easy release product (see Table of Materials) in a well-ventilated area. Assemble the lower half of myocardial case around the apex of the blood pool. If any seams between the myocardial case pieces allow the silicone to leak out, use clamps or a material such as hot glue or clay to seal the leak on the external surface of the mold.

5. Pour silicone into the space between the blood pool and case wall, allowing the silicone to flow into all gaps. Continue pouring silicone until the assembled pieces of the myocardial mold are filled with silicone.

6. Assemble the remaining pieces of the myocardial case, secure the case pieces tightly using rubber bands and clamps, as necessary. Pour silicone down the fill hole in the top of the myocardial case piece until the entire myocardial space is filled with silicone.

7. Allow the silicone to set for $\sim 40 \mathrm{~min}$. Remove the silicone heart from the myocardial case and trim off any silicone seams that were created from the space between the case pieces or the fill hole.

\section{Dissolve blood pool}

1. Identify all blood vessels that should have open ends on the silicone model and trim away any silicone that is covering them to expose the ABS blood pool inside.

2. Submerge the silicone heart in an acetone bath. ABS will begin to soften 10-15 min after acetone submersion; as this occurs, remove large chunks of ABS with tweezers to increase the speed of the ABS dissolving process. 
3. Perform two to three additional acetone rinses/soaks with clean acetone to remove all the ABS from the silicone when a majority of the ABS blood pool has dissolved. Remove the cardiac model from the acetone bath and allow the remaining acetone to evaporate from the model in a well-ventilated area. Time required to fully dissolve the ABS will depend upon the size of the model, the amount of $A B S$ removed manually, and the amount of acetone used.

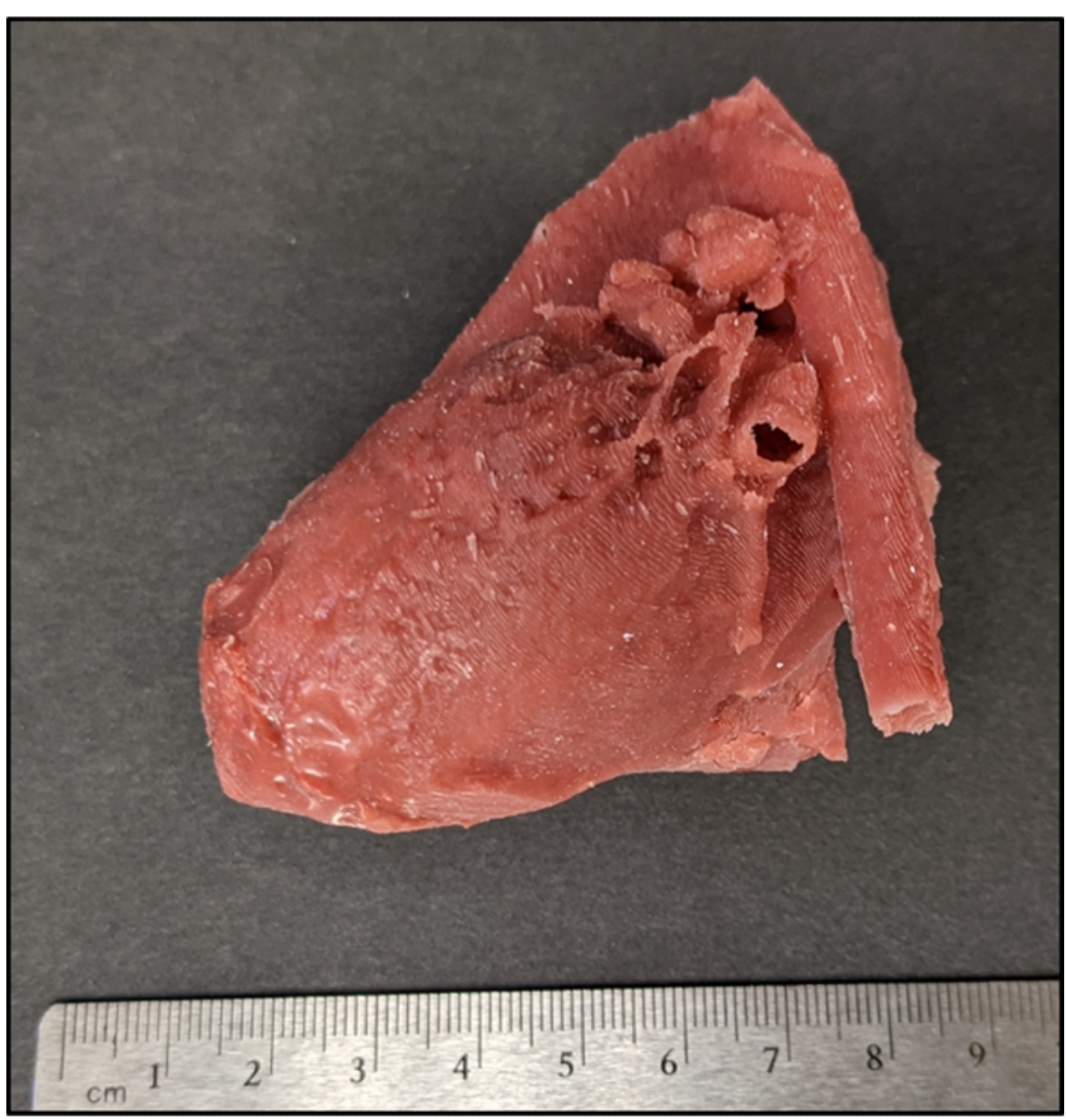

Figure 6: Patient-specific silicone cardiac model with a VSD. Photograph of an epicardial surface view of complete silicone model with a VSD. VSD is not visible due to its location within the intra-cardiac myocardial structure. Please click here to view a larger version of this figure.

\section{Representative Results}

Radiologic imaging data from a patient with a VSD was chosen to generate a representative silicone cardiac model. Patient anatomy was segmented utilizing a CAD segmentation software to generate a digital myocardial model and a digital blood pool model (Figure 1). Manual segmentation of blood pool and myocardium with the presented protocol takes 1-3 $\mathrm{h}$ to complete. Upon completion of segmentation, the myocardial model was opened in CAD 
software for further processing. The model was aligned to a 3D box made within the program and then subtracted away using Boolean operations. This process left a negative of the myocardial model, forming a mold. This myocardial mold was trimmed to a more appropriate size, cut into segments, and modified with props for aligning the pieces (Figure 2). The creation of the case took 2-6 h. All myocardial mold pieces and the blood pool were loaded in a $3 \mathrm{D}$ printing slicing software, and G-Code was generated to 3D print in ABS (Figure 3). The 3D printed pieces with support material removed can be seen in Figure 4. The myocardial case pieces were vapor smoothed to enhance the surface finish of the model (Figure 5). Upon the completion of the vapor smooth process, the mold was assembled around the blood pool model, and silicone was poured. The assembly and silicone pour took one hour. After the silicone set, the cardiac model was removed from the myocardial case and submerged in acetone to dissolve the blood pool. After approximately twenty-four hours of soaking, the blood pool had dissolved. A final acetone rinse was performed, and the model was allowed to fully dry. The completed silicone cardiac model can be seen in Figure 6. To evaluate the accuracy and functionality of the silicone model, an incision was made by the CHD (congenital heart defect) expert to allow the inner anatomy to be observed. The expected VSD was present, and a GORE-TEX patch was sewn onto the model by the congenital cardiac surgeon to correct the VSD (Figure 7). In a successfully completed silicone model, all patient anatomy and defects will be present both externally and internally. A summary of the protocol can be seen in Supplementary File 1.

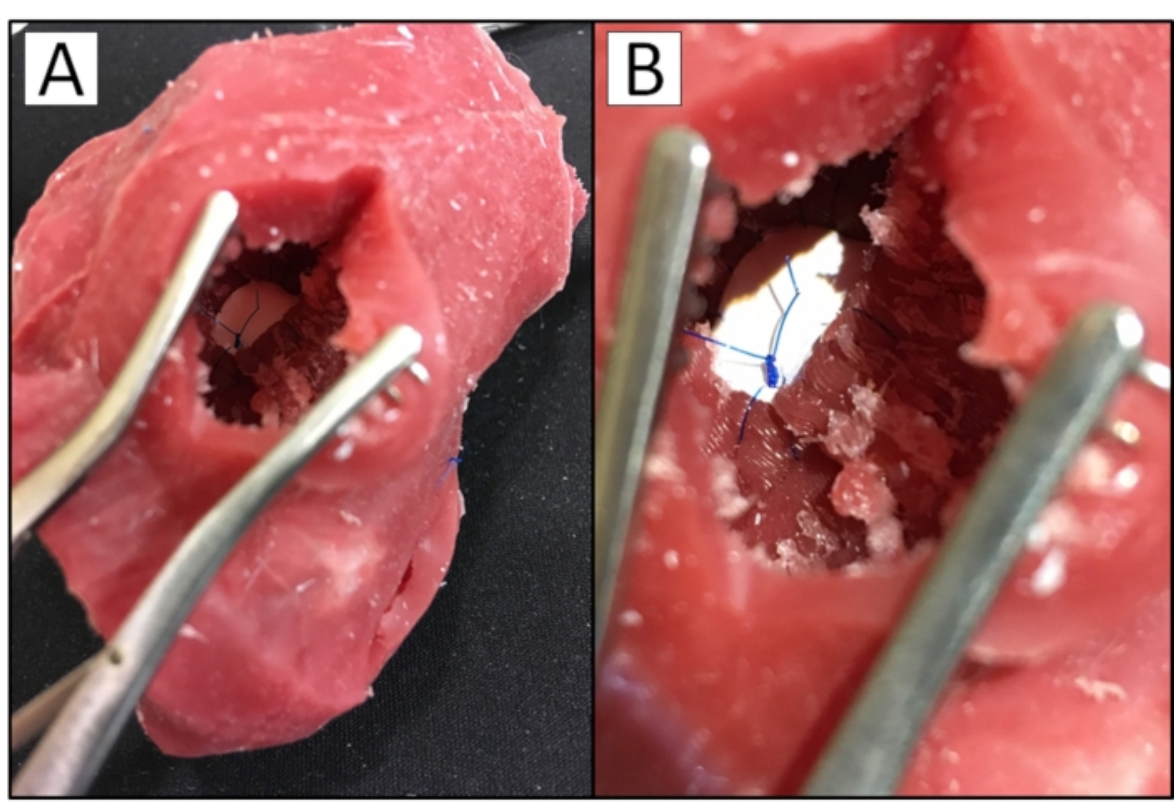

Figure 7: GORE-TEX patch sewn in silicone cardiac model with VSD. Photograph of (A) surgeon's view of a patientspecific silicone cardiac model with a VSD and (B) surgeon's view of the VSD in the model closed with a GORE-TEX patch. 


\section{Supplementary File 1: Schematic of silicone heart}

fabrication protocol. Schematic illustration of the protocol in the fabrication of a patient-specific silicone cardiac model. Please click here to download this File.

\section{Supplementary File 2: Summary of critical steps and} potential negative outcomes. Summary of the steps critical in the development of a patient-specific silicone cardiac model and the potential negative outcomes that can result if the steps are not followed correctly. Please click here to download this File.

\section{Discussion}

Upon completion of the protocol, a patient-specific silicone cardiac model for surgical preparation should result. However, there are several critical steps that must be completed correctly for this to be achieved. A summary of the critical steps in the protocol can be seen in Supplementary

File 2, as well as potential outcomes if the steps are not performed correctly. The first critical step involves the segmentation of the patient's radiologic imaging data. This step requires the acquisition of a diagnostic 3D imaging dataset. Model utility in pre-surgical planning or education is dependent on the quality of the 3D dataset. It is recommended to use an image set collected with a slice size between $0.625 \mathrm{~mm}$ and $2.6 \mathrm{~mm}$ to ensure the data set will be of adequate resolution for model production. However, all imaging parameters should be set by a clinician expert in radiology, with patient care being the priority. It should be noted that it may be possible to produce a model from an image set collected with a slice size outside the recommended values, but model resolution and quality will be negatively impacted. After images are obtained, if the segmentation is not performed correctly, it is commonly not realized until the final model is produced and cut into, resulting in a loss of time and materials. To prevent this negative outcome, it is recommended that a subject matter expert review the segmented files prior to creating the digital molds for quality control. The next critical step occurs during the creation of the digital molds. It is important to ensure that the myocardial case will be able to be assembled around the blood pool model. If the case does not close around the blood pool, it cannot be used to create the silicone model as unset silicone will continually leak out, and anatomy could be distorted. A handheld rotary sanding tool can be used to lightly remove pieces of the myocardial mold only if small adjustments are needed. If large adjustments are needed, the digital mold will need to be altered and an updated case printed. The final critical step is the pouring of the silicone. Strict adherence to material instructions is necessary when using the silicone, as failure to do so may result in silicone that cures with a tacky surface. If the surface is deemed too tacky for use by the SME, the blood pool may have to be reprinted if it cannot successfully be removed from the silicone. The silicone will have to be re-poured, resulting in a loss of time and materials. If insufficient silicone is used or the silicone leaks out of the myocardial case mold during the setting process, the resulting model will be incomplete. This failure can be remedied by mixing and pouring additional silicone into the mold. A material such as hot glue or clay can be used to seal the seams of the myocardial case mold if a small amount of silicone appears to be leaking through during the curing process.

This method of creating patient-specific silicone cardiac models can be modified to allow the creation of a model of any soft anatomical structure with patient-specific or complex inner and outer geometry. Assuming the target anatomy is segmented correctly, the remaining steps of the protocol could be followed with minimal change. While 
not the focus of the current work, the protocol has been applied to liver parenchyma with similar success. The utilized 3D print material can also be modified. ABS and PLA are recommended for use due to their low cost, but any dissolvable $3 \mathrm{D}$ print material can take the place of $\mathrm{ABS}$, and any desirable 3D print material can take the place of PLA with minimal or no change to the protocol. All filament manufacturer-specified printing parameters should be followed when using other print materials. This method can further be modified by the use of a different silicone. The silicone recommended for use in this protocol has a shore hardness of $2 \mathrm{~A}$, but if another shore hardness value is desirable, a different silicone can be substituted with minimal or no change to the protocol. Be sure to adhere to all manufacturing specifications and procedures when using a different silicone product.

While this protocol outlines an improved cardiac modeling procedure, it is not without limitations. The major limitation of this protocol is that while the utilized platinum cure silicone is closer to the hardness of cardiac tissue than other available materials, hardness is not the only property that plays a role in the fine-motor skill of surgical training. In particular, real cardiac tissue will demonstrate friability or tearing under force. The utilized silicone is very elastic, with an elongation at break of $763 \%$ and tensile strength of $1,986 \mathrm{kPa}^{19}$. Porcine cardiac tissue, which is believed to be an accurate representation of human cardiac tissue, has an elongation at break of $28-66 \%$ and tensile strength of $40-59 \mathrm{kPa}^{26}$. This difference presents a problem, as cardiac surgical fellows may perform a practice operation on a silicone model heart and gain a false sense of confidence because the model can withstand forces that real cardiac tissue cannot. This methodology also has the potential to be limited by a cardiac model with very complex geometry. As the anatomical complexity of the model increases, the protocol can compensate by increasing the number of pieces in the myocardial mold. Essentially, increasingly complex models will require increasingly complex mold designs and increased design time.

The model creation process described in this protocol is superior to many of the other available alternatives due to its ability to re-create low-cost exact anatomic replicas of surgically encountered anatomy. Cadaveric and animal tissue does allow for high fidelity simulations, but they have a much higher cost and require specific laboratory set-ups to be utilized and maintained ${ }^{2,6}$. Further, cadaveric and animal tissue models have ethical concerns, are not patient-specific, and complex $\mathrm{CHD}$ often must be manually manufactured by a surgeon or instructor, often leading to inaccuracies or damage to the surrounding tissues and organs. Another potential modeling technique involves the use of virtual reality. Virtual reality allows for the digital replication of patient-specific cardiac models, which is an effective tool for establishing accurate mental representations of patient anatomy and surgical plans. Additionally, some VR systems have allowed for basic simulations with the incorporation of haptic feedback. However, the available haptic feedback lacks the realism necessary to replicate necessary fine-motor skills for congenital heart surgical procedures ${ }^{4}$. 3D printing is another available method to produce patient-specific cardiac models $^{2,24}$. However, the widespread implementation of high-fidelity 3D printers capable of producing multi-material, soft models are inhibited by their extremely high $\operatorname{cost}^{11,14,15}$. Low-cost 3D printers are available but can only print in materials that are much firmer than real myocardium. When one of the softest available materials for a $3 D$ printer was used to create a model by Scanlan et al., the model was found to be firmer than real cardiac tissue ${ }^{17}$. The described 
material had a shore hardness between $26 \mathrm{~A}$ and $28 \mathrm{~A}$, giving it a texture similar to a rubber band. The platinum cured silicone used in this protocol has a shore hardness of $2 \mathrm{~A}$, giving it a texture similar to a gel shoe insert and much closer to the hardness of real cardiac tissues, which is $4300^{20}$ or $\sim 0 \mathrm{~A}$. Hoashi et al. also utilized a similar method to the one described in this protocol to develop a flexible 3D printed heart model. Two molds, representing the inner and outer myocardial geometry, were 3D printed utilizing an SLA printer followed by vacuum casting a rubberlike polyurethane resin. While this method did produce a soft cardiac model, the proposed production cost of this method per model was 2,000 to $3,000 U_{S D}^{22}$. Comparatively, the total material cost of the method described in the presented protocol is less than 10 USD. Finally, a similar method was also used by Russo et al. to create silicone models of the aortic valve and proximal aorta for procedural practice. While the Russo et al. method is focused on a similar goal, their presented process aimed at replicating far simpler anatomies of the aorta or aortic valves. The protocol presented herein differentiates itself by focusing on intracardiac and myocardial anatomies that are smaller, more complex, and would be extremely difficult to replicate given historical methodologies. Despite this difference, the models created by Russo et al. were highly useful for simulation and training in cardiac surgery by surveyed cardiac surgeons ${ }^{23}$. Essentially, the method described in this protocol allows for the low-cost creation of complex, patient-specific congenital cardiac models with accurately represented defects and material properties more similar to real cardiac tissue than other modeling methods ${ }^{1,16}$, allowing models to be operated on with a realistic haptic fidelity.

Moving forward, this methodology could be applied to the formation of a model of any patient anatomy with complex internal and external features. Developing an alternative blood pool material that could be removed from within the silicone model in a less destructive manner or produced using a less time-consuming method would make the process more time- and cost-efficient. As a result, a new blood pool would not have to be reproduced for each subsequent molding process, leading to the scalability of the associated training. The physical properties of the silicone used to create the model could also be improved. Silicone with less elongation at break would increase the realism of the model and help to improve its value as an educational tool for cardiac surgical fellows trying to learn the necessary fine motor skills for performing these complex procedures. A group of materials currently on the market worthy of consideration to aid in this solution are silicone simulated glass materials ${ }^{25}$. These silicone materials demonstrate much less elongation at break leading to a distinct "shattering" upon force application in a manner similar to glass. Modulating the platinum cure silicone used in this protocol with additions of this silicone simulated glass material may allow for control of the friability characteristics of the model while still maintaining the appropriate shore hardness, improving the overall haptic fidelity. Finally, the resolution of anatomy this protocol can produce is limited by the resolution of the 3D printer utilized to generate the molds. As technology continues to improve, the resolution of anatomy that can be created with this protocol should also improve.

\section{Disclosures}

The authors have nothing to disclose.

\section{Acknowledgments}

The authors would like to acknowledge OSF HealthCare for making this study possible, as well as Dr. Mark Plunkett for 
his procedural knowledge and application of skills to our final product.

\section{References}

1. Hoffman, J. I. E., Kaplan, S. The incidence of congenital heart disease. Journal of the American College of Cardiology. 39 (12) 1890-1900 (2002).

2. Hussein, N., et al. Hands-on surgical simulation in congenital heart surgery: Literature review and future perspective. Seminars in Thoracic and Cardiovascular Surgery. 32 (1), 98-105 (2020).

3. Wilson, H. K., Feins, R. H. Simulation in cardiothoracic surgery. Comprehensive Healthcare Simulation: Surgery and Surgical Subspecialties. Comprehensive Healthcare Simulation. 263-274, Springer, Cham (2019).

4. Badash, I., Burtt, K., Solorzano, C. A., Carey, J. Innovations in surgery simulation: A review of past, current and future techniques. Annals of Translational Medicine. 4 (23), 1-10 (2016).

5. Yoo, S. J., Spray, T, Austin, E. H., Yun, T. J., Van Arsdell, G. S. Hands-on surgical training of congenital heart surgery using 3-dimensional print models. Journal of Thoracic and Cardiovascular Surgery. 153, 1530-1540 (2017).

6. Hadeed, K., Acar, P., Karsenty, C. Cardiac 3D printing for better understanding of congenital heart disease. Archives of Cardiovascular Disease. 111 (1), 1-4 (2018).

7. Velasco Forte, M. N., et al. Living the heart in three dimensions: applications of 3D printing in CHD. Cardiology in the Young. 29, 733-743 (2019).

8. Illmann, C. F., Ghadiry-Tavi, R., Hosking, M., Harris, K. C. Utility of 3D printed cardiac models in congenital heart disease: a scoping review. Heart. 106, 1631-1637 (2020).

9. Su, W., Xiao, Y., He, S., Huang, P., Deng, X. Threedimensional printing models in congenital heart disease education for medical students: a controlled comparative study. BMC Medical Education. 18 (178) (2018).

10. Farooqi, K. M., Mahmood, F. Innovations in preoperative planning: insights into another dimension using 3D printing for cardiac disease. Journal of Cardiothoracic and Vascular Anesthesia. 32, 1937-1945 (2018).

11. Illmann, C. F., Hosking, M., Harris, K. C. Utility and access to 3-dimensional printing in the context of congenital heart disease: an international physician survey study. Canadian Cardiovascular Society. 2, 207-213 (2020).

12. Lau, I., Gupta, A., Sun, Z. Clinical value of virtual reality versus $3 \mathrm{D}$ printing in congenital heart disease. Biomolecules. 11 (884) (2021).

13. Birbara, N. S., Otton, J. M., Pather, N. 3D modelling and printing technology to produce patient-specific 3D models. Heart Lung and Circulation. 28, 302-313 (2019).

14. Yoo, S. J., et al. 3D printing in medicine of congenital heart disease. 3D Printing in Medicine. 2 (3) (2016).

15. Gómez-Ciriza, G., Gómez-Cía T., Rivas-González J. A., Velasco Forte, M. N., Valverde, I. Affordable three-dimensional printed heart models. Frontiers in Cardiovascular Medicine. 8, 498 (2021).

16. Lau, I., et al. Quantitative and qualitative comparison of low- and high-cost 3D-printed heart models. Quantitative Imaging in Medicine and Surgery. 9 (1), 107-114 (2019).

17. Scanlan, A. B. et al. Comparison of 3D echocardiogram derived $3 \mathrm{D}$ printed valve models to molded models 
for simulated repair of pediatric atrioventricular valves. Pediatric Cardiology. 39 (3), 538-547 (2019).

18. Tango polyJet simulated rubber material, Statasys. at $<$ www.stratasys.com> (2018).

19. Dragon skin fx-pro, Smooth-On. at <https://www.smoothon.com/products/dragon-skin-fx-pro/> (2021).

20. Tejo-Otero, A., Fenollosa-Artés, F., Buj-Corral, I. Mimicking soft living tissues for 3D printed surgical planning prototypes using different materials. Congreso Anual de la Sociedad Española de Ingeniería Biomédica. 307-310 (2019).

21. Lezhnev, A. A., Ryabtsev, D. V., Hamanturov, D. B., Barskiy, V. I., Yatsyk, A. P. Silicone models of the aortic root to plan and simulate interventions. Interactive CardioVascular and Thoracic Surgery. 31 (2), 204-209 (2020).

22. Laing, J. A patient-specific cardiac phantom for training and pre-procedure surgical planning. Electronic Thesis and Dissertation Repository. 4964 (2017).

23. Hoashi, T., et al. Utility of a super-flexible threedimensional printed heart model in congenital heart surgery. Interactive CardioVascular and Thoracic Surgery. 27, 749-755 (2018).

24. Mena, K. A., et al. Exploration of time-sequential, patientspecific 3D heart unlocks clinical understanding. 3D Printing in Medicine. 4 (1), 15 (2018).

25. Russo, M., et al. Advanced three-dimensionally engineered simulation model for aortic valve and proximal aorta procedures. Interactive CardioVascular and Thoracic Surgery. 30, 887-895 (2020).

26. Al Ali, A. B., Griffin, M. F., Butler, P. E. Three-dimensional printing surgical applications. ePlasty. 15, e37 (2015).
27. Rubber glass water-clear silicone rubber compound, Smooth-On. at <https://www.smooth-on.com/productline/rubber-glass/> (2021).

28. Riedle, H., Molz, P., Franke, J., Determination of the mechanical properties of cardiac tissue for 3D printed surgical models. IEEE-EMBS Conference on Biomedical Engineering and Science. 171-176 (2018). 